\title{
Quadratic polynomial maps with Jacobian rank two
}

\author{
Michiel de Bondt* \\ Institute for Mathematics, Astrophisics and Particle Physics \\ Radboud University Nijmegen \\ Email address: M.deBondt@math.ru.nl
}

June 20, 2018

\begin{abstract}
Let $K$ be any field and $x=\left(x_{1}, x_{2}, \ldots, x_{n}\right)$. We classify all matrices $M \in \operatorname{Mat}_{m, n}(K[x])$ whose entries are polynomials of degree at most 1 , for which $\operatorname{rk} M \leq 2$. As a special case, we describe all such matrices $M$, which are the Jacobian matrix $\mathcal{J} H$ (the matrix of partial derivatives) of a polynomial map $H$ from $K^{n}$ to $K^{m}$.

Among other things, we show that up to composition with linear maps over $K, M=\mathcal{J} H$ has only two nonzero columns or only three nonzero rows in this case. In addition, we show that $\operatorname{trdeg}_{K} K(H)=\operatorname{rk} \mathcal{J} H$ for quadratic polynomial maps $H$ over $K$ such that $\frac{1}{2} \in K$ and rk $\mathcal{J} H \leq 2$.

Furthermore, we prove that up to conjugation with linear maps over $K$, nilpotent Jacobian matrices $N$ of quadratic polynomial maps, for which rk $N \leq 2$, are triangular (with zeroes on the diagonal), regardless of the characteristic of $K$. This generalizes several results by others.

In addition, we prove the same result for Jacobian matrices $N$ of quadratic polynomial maps, for which $N^{2}=0$. This generalizes a result by others, namely the case where $\frac{1}{2} \in K$ and $N(0)=0$.
\end{abstract}

Key words: quadratic polynomial map, Jacobian rank two, transcendence degree two, homogeneous, nilpotent, unipotent Keller map, linearly triangularizable, strongly nilpotent, equivalent over $K$, similar over $K$.

MSC 2010: 12E05, 12F20, 14R05, 14R10.

\footnotetext{
${ }^{*}$ The author was supported by the Netherlands Organisation for Scientific Research (NWO).
} 


\section{Introduction}

Throughout this paper, $K$ is an arbitrary field and $x:=\left(x_{1}, x_{2}, \ldots, x_{n}\right)$ is an $n$-tuple of indeterminates. We write $\left.a\right|_{b=c}$ for the result of substituting $b$ by $c$ in $a$.

We call a polynomial $h \in K[x]$ homogeneous (of degree $d$ ) if all terms of $h$ have the same degree (and $\operatorname{deg} h=d$ ). We call the terms of degree $\operatorname{deg} h$ of $h$ the leading homogeneous part of $h$. A linear form is a polynomial $h \in K[x]$ which is homogeneous of degree 1.

Let $H \in K[x]^{m}$. Then $H=\left(H_{1}, H_{2}, \ldots, H_{m}\right)$ is a polynomial map from $K^{n}$ to $K^{m}$. The degree of $H$ is defined by $\operatorname{deg} H:=\max \left\{\operatorname{deg} H_{1}, \operatorname{deg} H_{2}, \ldots\right.$, $\left.\operatorname{deg} H_{m}\right\}$. We say that $H$ is homogeneous (of degree $d$ ) if $y_{1} H_{1}+y_{2} H_{2}+\cdots+$ $y_{m} H_{m}$ is a homogeneous polynomial (of degree $d+1$ ).

We write $\mathcal{J} H$ for the Jacobian matrix of $H$ (with respect to $x$ ), i.e.

$$
\mathcal{J} H=\left(\begin{array}{cccc}
\frac{\partial}{\partial x_{1}} H_{1} & \frac{\partial}{\partial x_{2}} H_{1} & \cdots & \frac{\partial}{\partial x_{n}} H_{1} \\
\frac{\partial}{\partial x_{1}} H_{2} & \frac{\partial}{\partial x_{2}} H_{2} & \cdots & \frac{\partial}{\partial x_{n}} H_{2} \\
\vdots & \vdots & \vdots \vdots & \vdots \\
\frac{\partial}{\partial x_{1}} H_{m} & \frac{\partial}{\partial x_{2}} H_{m} & \cdots & \frac{\partial}{\partial x_{n}} H_{m}
\end{array}\right)
$$

We call a matrix a Jacobian matrix if it is the Jacobian matrix of some polynomial map. Note that this polynomial map is not uniquely determined if our base field $K$ has positive characteristic.

Let $R$ be a commutative ring with 1 . We write $\operatorname{Mat}_{m, n}(R)$ for the abelian group of matrices with $m$ rows and $n$ columns over $R$. So $\mathcal{J} H \in \operatorname{Mat}_{m, n}(K[x])$. We write $\operatorname{Mat}_{n}(R)$ for the ring of matrices with $n$ rows and $n$ columns over $R$. We define $\mathrm{GL}_{n}(R)$ as the group of invertible matrices in $\operatorname{Mat}_{n}(R)$, i.e. $\mathrm{GL}_{n}(R):=\left\{M \in \operatorname{Mat}_{n}(R) \mid \operatorname{det} M\right.$ is a unit in $\left.R\right\}$.

If $R$ is a $K$-algebra, then we say that elements $M$ and $\tilde{M}$ of $\operatorname{Mat}_{m, n}(R)$ are equivalent over $K$ if there exists matrices $S \in \mathrm{GL}_{m}(K)$ and $T \in \mathrm{GL}_{n}(K)$ such that $\tilde{M}=S M T$. If $m=n$ and $S=T^{-1}$ in addition, then we say that $M$ and $\tilde{M}$ are similar over $K$.

A matrix $M \in \operatorname{Mat}_{n}(R)$ is upper (lower) triangular if all entries below (above) the principal diagonal are zero, and triangular (diagonal) if $M$ is either (both) upper or (and) lower triangular. So a diagonal matrix may only have nonzero entries on the diagonal which runs from its upper left corner to its lower right corner. This diagonal is called the (principal) diagonal.

We say that a matrix $M \in \operatorname{Mat}_{n}(R)$ is nilpotent if the exists an $r \geq 1$, such that $M^{r}=0$. The reader may verify the following.

Lemma 1.1. Suppose that $R$ is a $K$-algebra and $M \in \operatorname{Mat}_{n}(R)$.

(i) If $M$ is upper (lower) triangular, then $M$ is similar over $K$ to a lower (upper) triangular matrix $\tilde{M} \in \operatorname{Mat}_{n}(R)$

(ii) If $\tilde{M}$ is similar over $K$ to $M$, then $\tilde{M}$ is nilpotent, if and only if $M$ is nilpotent. 
If $M \in \operatorname{Mat}_{m, n}(K[x])$, then we write $M(v)$ for the matrix

$$
\left(\begin{array}{cccc}
M_{11}(v) & M_{12}(v) & \cdots & M_{1 n}(v) \\
M_{21}(v) & M_{22}(v) & \cdots & M_{2 n}(v) \\
\vdots & \vdots & \vdots \vdots & \vdots \\
M_{m 1}(v) & M_{m 2}(v) & \cdots & M_{m n}(v)
\end{array}\right)
$$

where $v \in K^{n}$. We say that $M \in \operatorname{Mat}_{m}(K[x])$ is strongly nilpotent (over $K$ ) if there exists an $r \geq 1$, such that

$$
M\left(v^{(1)}\right) \cdot M\left(v^{(2)}\right) \cdots M\left(v^{(r)}\right)=0
$$

for all $v^{(1)}, v^{(2)}, \ldots, v^{(r)} \in K^{n}$. If $K$ is infinite, then proposition 1.3 below gives a classification of strongly nilpotent matrices over $K[x]$. For the proof of proposition 1.3, we need the following lemma, which one can show by induction on $r$.

Lemma 1.2. Suppose that $M \in \operatorname{Mat}_{m}(K[x])$ is of the form

$$
\left(\begin{array}{cc}
A & \theta \\
* & B
\end{array}\right) \quad \text { or } \quad\left(\begin{array}{cc}
A & * \\
\theta & B
\end{array}\right)
$$

Let $\tilde{M}:=M\left(v^{(1)}\right) \cdot M\left(v^{(2)}\right) \cdots M\left(v^{(r)}\right), \tilde{A}:=A\left(v^{(1)}\right) \cdot A\left(v^{(2)}\right) \cdots \cdot A\left(v^{(r)}\right)$ and $\tilde{B}:=B\left(v^{(1)}\right) \cdot B\left(v^{(2)}\right) \cdots B\left(v^{(r)}\right)$, where $v^{(i)} \in K[x]^{n}$ for each $i$. Then $\tilde{M}$ is of the form.

$$
\left(\begin{array}{cc}
\tilde{A} & \theta \\
* & \tilde{B}
\end{array}\right) \quad \text { or } \quad\left(\begin{array}{cc}
\tilde{A} & * \\
\theta & \tilde{B}
\end{array}\right)
$$

respectively.

Proposition 1.3. Suppose that $L$ is infinite and an extension field of $K$. Let $M \in \operatorname{Mat}_{m}(K[x])$. Then $M$ is strongly nilpotent over $L$, if and only if $M$ is similar over $K$ to a triangular matrix in $\operatorname{Mat}_{m}(K[x])$, whose principal diagonal is totally zero.

Proof. The 'if'-part is an straightforward exercise, so assume that $M$ is strongly nilpotent over $L$. Then there are $v^{(2)}, v^{(3)}, \ldots, v^{(r)} \in L^{n}$ such that for all $v^{(1)} \in$ $L^{n}$,

$$
M\left(v^{(1)}\right) \cdot M\left(v^{(2)}\right) \cdots \cdot M\left(v^{(r)}\right)=0 \neq M\left(v^{(2)}\right) \cdot M\left(v^{(3)}\right) \cdots M\left(v^{(r)}\right)
$$

Since $L$ is infinite, it follows that

$$
M \cdot M\left(v^{(2)}\right) \cdots M\left(v^{(r)}\right)=0 \neq M\left(v^{(2)}\right) \cdot M\left(v^{(3)}\right) \cdots M\left(v^{(r)}\right)
$$

so the columns of $M$ are linearly dependent over $L$. Since $L$ is a vector space over $K$, the columns of $M$ are linearly dependent over $K$. Hence $M$ is similar over $K$ to a matrix $\tilde{M} \in \operatorname{Mat}_{m}(K[x])$, of which the last column is zero.

From lemma 1.2, it follows that the upper left submatrix of size $(m-1) \times$ $(m-1)$ of $\tilde{M}$ strongly nilpotent. By induction on $m$, it follows that $\tilde{M}$ and hence also $M$ is similar over $K$ to a lower triangular matrix in $\operatorname{Mat}_{m}(K[x])$, whose principal diagonal is totally zero. 
The above proof has been extracted from that of [dB1, Th. 3.1], which is a more general result.

Corollary 1.4. Suppose that $M \in \operatorname{Mat}_{m}(K[x])$ is of the form

$$
\left(\begin{array}{cc}
A & \theta \\
* & B
\end{array}\right) \quad \text { or } \quad\left(\begin{array}{cc}
A & * \\
\theta & B
\end{array}\right)
$$

Then $M$ is similar over $K$ to a triangular matrix with only zeroes on its principal diagonal, if and only if $A$ and $B$ are similar over $K$ to triangular matrices with only zeroes on their principal diagonals.

Proof. From lemma 1.2 it follows that $A$ and $B$ are strongly nilpotent over an infinite extension field $L$ of $K$ if $M$ is strongly nilpotent over $L$. Hence the 'only if'-part follows from proposition 1.3 .

To prove the 'if'-part, suppose that $A$ and $B$ are strongly nilpotent over an infinite extension field $L$. Then there exists an integer $r$, such that $\tilde{A}=\tilde{B}=0$ in (1.1), for every $v^{(1)}, v^{(2)}, \ldots, v^{(r)} \in L^{n}$. It follows that

$$
\left(M\left(v^{(1)}\right) \cdot M\left(v^{(2)}\right) \cdots M\left(v^{(r)}\right)\right) \cdot\left(M\left(v^{(r+1)}\right) \cdot M\left(v^{(r+2)}\right) \cdots M\left(v^{(2 r)}\right)\right)=0
$$

for all $v^{(1)}, v^{(2)}, v^{(3)}, \ldots, v^{(2 r-1)}, v^{(2 r)} \in K^{n}$. Hence the 'if'-part follows from proposition 1.3 as well.

A minor (determinant) of a matrix $M$ is the determinant of a square submatrix, say $N$, of $M$. The submatrix $N$ itself is called a minor matrix. If the entries of the (principal) diagonal of $N$ lie on the (principal) diagonal of $M$ as well, then $\operatorname{det} N$ and $N$ are called a principal minor (determinant) and a principal minor matrix respectively.

Notice that a minor matrix $N$ of $M$ is a principal minor matrix of $M$, if and only if the indices of the rows of $M$ which $N$ has as a submatrix of $M$ are the same as the indices of the columns of $M$ which $N$ has as a submatrix.

We call a principal minor matrix of $M$ a leading principal minor matrix if it extends from the upper left corner of $M$, and a trailing principal minor matrix if it extends from the lower right corner of $M$. Similarly, we define leading principal minor (determinant) and trailing principal minor (determinant).

We call $\lambda$ an eigenvalue of $M \in \operatorname{Mat}_{m}(K)$ if there exists a nonzero $v \in K^{m}$ such that $M v=\lambda v$. Notice that we must view the vector $v$ as a matrix with only one column in $M v=\lambda v$. We will regard vectors as matrices with only one column in the rest of the paper as well.

Lemma 1.5. Suppose that $M \in \operatorname{Mat}_{m}(K)$. Then the following statements are equivalent.

(1) $M$ is nilpotent;

(2) every eigenvalue of $M$ equals zero;

(3) for every $r \leq m$, the sum of the principal minor determinants of size $r \times r$ is zero. 
Proof.

(1) $\Rightarrow$ (2) Suppose that $\lambda$ is an eigenvalue of $M$. Then $M$ is similar over $K$ to a matrix $\tilde{M}$ for which $\tilde{M} e_{1}=\lambda e_{1}$. Since $\tilde{M}$ is nilpotent as well, it follows from lemma 1.2 that $\lambda=0$.

(2) $\Rightarrow$ (3) Let $f(u)=\operatorname{det}\left(u I_{m}+M\right)$. Then for every $r$ for which $1 \leq r \leq$ $m$, the coefficient of $u^{m-r}$ in $f$ equals the sum of the principal minor determinants of size $r \times r$ of $M$.

If $f(-\lambda)=0$, then $\operatorname{ker}\left(-\lambda I_{m}+M\right) \neq\{0\}$, and $\lambda$ is an eigenvalue of $M$. Suppose that (2) holds. Then $u=-0$ is the only root of $f$. Hence $f=u^{m}$ and (3) follows.

(3) $\Rightarrow$ (1) Suppose that (3) holds. Then $f=u^{m}$. From the Cayley-Hamilton theorem, it follows that $(-M)^{m}=0$, which gives (1).

Suppose that $M=\mathcal{J} H$ and $\tilde{M}=S M T$, where $H$ is a polynomial map from $K^{n}$ to $K^{m}, S \in \mathrm{GL}_{m}(K)$ and $T \in \mathrm{GL}_{n}(K)$. Let $\tilde{H}:=S H(T x)$. From the chain rule, it follows that

$$
\mathcal{J} \tilde{H}=\mathcal{J}(S H(T x))=\left.S M\right|_{x=T x} T=\left.\tilde{M}\right|_{x=T x}
$$

so $\tilde{M}$ itself is a Jacobian matrix up to an automorphism of $K[x]$. It follows that $\mathcal{J} \tilde{H}$ is (strongly) nilpotent or upper (lower) triangular, if and only if $\tilde{M}$ is (strongly) nilpotent or upper (lower) triangular respectively.

The degree of a matrix $M \in \operatorname{Mat}_{m, n}(K[x])$ is defined by

$\operatorname{deg} M:=\max \left\{\operatorname{deg} M_{11}, \operatorname{deg} M_{12}, \ldots, \operatorname{deg} M_{1 n}, \operatorname{deg} M_{21}, \operatorname{deg} M_{22}, \ldots, \operatorname{deg} M_{m n}\right\}$

and we write $\operatorname{rk} M$ for the rank of $M$.

Let $M \in \operatorname{Mat}_{m, n}(R)$, where $R$ is a ring. Then we denote by $M^{\mathrm{t}}$ the transpose of $M$, so

$$
M^{\mathrm{t}}=\left(\begin{array}{cccc}
M_{11} & M_{12} & \cdots & M_{1 n} \\
M_{21} & M_{22} & \cdots & M_{2 n} \\
\vdots & \vdots & \vdots: \vdots & \vdots \\
M_{m 1} & M_{m 2} & \cdots & M_{m n}
\end{array}\right)^{\mathrm{t}}:=\left(\begin{array}{cccc}
M_{11} & M_{21} & \cdots & M_{m 1} \\
M_{12} & M_{22} & \cdots & M_{m 2} \\
\vdots & \vdots & \vdots \vdots & \vdots \\
M_{1 n} & M_{2 n} & \cdots & M_{m n}
\end{array}\right)
$$

Notice that the symbol $\mathrm{t}$ is upright to distinguish from taking the $t^{\text {th }}$ power. We call $M$ symmetric if $M^{\mathrm{t}}=M$ and antisymmetric if $M^{\mathrm{t}}=-M$.

Suppose that $K$ is a subfield of a field $L$. Then we write $\operatorname{trdeg}_{K} L$ for the transcendence degree of $L$ over $K$, i.e.

$\operatorname{trdeg}_{K} L=\max \{\# S \mid S \subseteq L$ and $S$ is algebraically independent over $K\}$

Here, a subset $S$ of $L$ is algebraically independent over $K$ if the result of substituting elements of $S$ in a nonzero polynomial in finitely many variables over $K$ will never be zero. 
§2 In section 2, we classify all matrices $M \in \operatorname{Mat}_{m, n}(K[x])$ for which $\operatorname{deg} M=$ 1 and $\operatorname{rk} M \leq 2$. Furthermore, we classify all such matrices $M$ such that $M=$ $\mathcal{J} H$ for some polynomial map $H$. Among other things, we show that $M=\mathcal{J} H$ is equivalent over $K$ to a matrix $\tilde{M}$ which has either only two nonzero columns or only three nonzero rows.

In addition, we show that $\operatorname{rk} \mathcal{J} H=\operatorname{trdeg}_{K} K(H)$ for quadratic polynomial maps $H$ over $K$ such that $\frac{1}{2} \in K$ and $\operatorname{rk} \mathcal{J} H \leq 2$. In general $\operatorname{rk} \mathcal{J} H \leq$ $\operatorname{trdeg}_{K} K(H)$ for a rational map $H$ of any degree, with equality if $K(H) \subseteq K(x)$ is separable, in particular if $K$ has characteristic zero. This is proved in dB3, Th. 1.3], see also [PSS, Ths. 10, 13].

\$3 In section 3, we prove that nilpotent Jacobian matrices $N$ for which $\operatorname{deg} N=$ 1 and $\operatorname{rk} N \leq 2$ are similar over $K$ to a triangular matrix (with zeroes on the diagonal), regardless of the characteristic of $K$. This generalizes [BY, Th. 3.4] (the case where $K$ has characteristic zero) and [PC, Th. 1] (the case where $\frac{1}{2} \in K$ and $\left.N(0)=0\right)$. In [PC, Th. 1], which is the main result of [PC], the authors additionally assume that $K$ is infinite, but one can derive the finite case from the infinite case by way of proposition 1.3 above.

At the end of section 3, we prove that nilpotent Jacobian matrices $N$ for which $\operatorname{deg} N=1$ and $N^{2}=0$ are similar over $K$ to a triangular matrix (with zeroes on the diagonal), regardless of the characteristic of $K$. This generalizes [MO, §4] and [PC, Lm. 4] (the case where $\frac{1}{2} \in K$ and $N(0)=0$ ).

We additionally show that $N\left(v^{(1)}\right) \cdot N\left(v^{(2)}\right) \cdot N\left(v^{(3)}\right)=0$ if $\frac{1}{2} \in K$, where $v^{(1)}, v^{(2)}, v^{(3)}$ are as in the proof of proposition 1.3. using the fact that the proof of [PC, Lm. 4] shows that $N\left(v^{(1)}\right) \cdot N\left(v^{(2)}\right)=0$ if $N(0)=0$ in addition.

\section{2 (Jacobian) matrices of degree one and rank at most two}

A matrix of rank zero can only be the zero matrix, so we only need to distinguish rank one and rank two. Let us start with rank one.

Theorem 2.1. Let $M$ be a matrix whose entries are polynomials of degree at most 1 over $K$. If $\operatorname{rk} M=1$, then $M$ is equivalent over $K$ to a matrix $\tilde{M}$ for which one of the following statements holds.

(1) Only the first column of $\tilde{M}$ is nonzero.

(2) Only the first row of $\tilde{M}$ is nonzero.

If $M$ is the Jacobian matrix of a (quadratic) polynomial map in addition, then the following assertion can be added to (1).

(1) The first column of $\tilde{M}$ is of the form $\left(*, \frac{1}{2}, 0, \ldots, 0\right)$. 
Proof. If the constant part $M(0)$ of $M$ is zero, then we can replace $M$ by the result of substituting $x_{i}=x_{i}+1$ in $M$, where $x_{i}$ is an indeterminate which appears in $M$, to obtain $M(0) \neq 1$. So we may assume that $M(0) \neq 0$.

Since $\operatorname{rk} M(0)=1$, we can choose $\tilde{M}$ such that

$$
\tilde{M}(0)=\left(\begin{array}{cccc}
1 & 0 & 0 & \ldots \\
0 & 0 & 0 & \ldots \\
0 & 0 & 0 & \ldots \\
\vdots & \vdots & \vdots & \vdots \vdots
\end{array}\right)
$$

Looking at the linear parts of the $2 \times 2$ minor determinants, we see that only the first row and the first column of $\tilde{M}$ may be nonzero.

Suppose that (1) does not hold. Then we may assume that the second entry of the first row of $M$ is nonzero. Suppose that (2) does not hold. Then we may assume that the first entry of the second row of $\tilde{M}$ is nonzero. This contradicts that the leading principal $2 \times 2$ minor determinant of $\tilde{M}$ is zero.

So we have proved the first part of this theorem. To prove the second part of this theorem, assume that $M=\mathcal{J} H$ for a polynomial map $H$. If we remove terms $x_{1}^{k_{1}} x_{2}^{k_{2}} \cdots$ of $H$ for which $\frac{1}{k_{i}} \notin K$ for all $i$, then $M=\mathcal{J} H$ is preserved, and for every term $t$ of $H$, there exists an $i$ such that

$$
\frac{\partial}{\partial x_{i}} t \neq 0
$$

Since $\operatorname{deg} \mathcal{J} H \leq 1$, it follows that $H$ becomes a polynomial map with terms of degree 1 and 2 only. So we may assume that $H(0)=0$ and $\operatorname{deg} H \leq 2$.

Say that $\tilde{M}=S M T$ for invertible matrices $S, T$ over $K$. Let $\tilde{H}:=S H(T x)$ and suppose that $\tilde{M}$ is as in (1). From (1.2), it follows that $\mathcal{J} \tilde{H}$ is as in (1) as well, i.e. only the first column of $\mathcal{J} \tilde{H}$ is nonzero.

If $\frac{1}{2} \in K$, then for all $j, \tilde{H}_{j}$ is linearly dependent over $K$ on $x_{1}^{2}$ and $x_{1}$ only. If $x_{1}$ and $x_{1}^{2}$ are in turn linearly dependent over $K$ on $\tilde{H}_{1}, \tilde{H}_{2}, \cdots$, then we can get the first column of $\mathcal{J} \tilde{H}$ and $\tilde{M}$ of the given form by way of row operations. Otherwise, we can get the first column of $\mathcal{J} \tilde{H}$ and $\tilde{M}$ of the form $(*, 0,0, \cdots, 0)$ by way of row operations, so (2) is satisfied.

So assume that $\frac{1}{2} \notin K$. Then for all $j, \tilde{H}_{j}$ is dependent over $K$ on $x_{1}, x_{1}^{2}, x_{2}^{2}$, $x_{3}^{2}, \ldots$. Hence the first column of $\mathcal{J} \tilde{H}$ is constant. By way of row operations, we can get the first column of $\mathcal{J} \tilde{H}$ and $\tilde{M}$ of the form $(*, 0,0, \cdots, 0)$. Hence only the first row of $\mathcal{J} \tilde{H}$ is nonzero and $\tilde{M}$ is as in (2).

In dB2, Th. 1.8], it is proved that over fields of characteristic zero, polynomial maps with an antisymmetric Jacobian matrix are linear. With essentially the same proof, one can draw the same conclusion if the characteristic of the field exceeds the degree of the polynomial map.

Lemma 2.2. Let $H$ be a polynomial map of degree at most d over $K$, such that $d ! \neq 0$ in $K$. If $\mathcal{J} H$ is antisymmetric, then $\operatorname{deg} H \leq 1$. 
Proof. There is nothing to prove if $\frac{1}{2} \notin K$, so assume that $\frac{1}{2} \in K$. Suppose that $\mathcal{J} H$ is antisymmetric. Then

$$
\frac{\partial}{\partial x_{i}} \frac{\partial}{\partial x_{j}} H_{k}=-\frac{\partial}{\partial x_{i}} \frac{\partial}{\partial x_{k}} H_{j}=\frac{\partial}{\partial x_{j}} \frac{\partial}{\partial x_{k}} H_{i}=-\frac{\partial}{\partial x_{i}} \frac{\partial}{\partial x_{j}} H_{k}
$$

and hence $2 \frac{\partial}{\partial x_{i}} \frac{\partial}{\partial x_{j}} H_{k}=0$, for all $i, j, k$. As $2 d ! \neq 0$ in $K$, it follows that $\operatorname{deg} H \leq 1$.

Using lemma 2.2 above, we can proceed with rank two.

Theorem 2.3. Let $M$ be a matrix whose entries are polynomials of degree at most 1 over $K$. If $\operatorname{rk} M=2$, then $M$ is equivalent over $K$ to a matrix $\tilde{M}$ for which one of the following statements holds.

(1) Only the first two columns of $\tilde{M}$ are nonzero.

(2) Only the first two rows of $\tilde{M}$ are nonzero.

(3) The first row and the first column of $\tilde{M}$ are nonzero, and $\tilde{M}$ is zero elsewhere.

(4) The leading principal $3 \times 3$ minor matrix of $\tilde{M}$ is anti-symmetric, with only zeroes on the diagonal, and $\tilde{M}$ is zero elsewhere. Furthermore, the three entries below the diagonal of this principal minor matrix are linearly independent over $K$.

If $M$ is the Jacobian matrix of a (quadratic) polynomial map in addition, then the following assertions can be added to (3) and (4) respectively.

(3) The first column of $\tilde{M}$ is of the form $\left(*, *, \frac{1}{2}, 0, \ldots, 0\right)$.

(4) $\tilde{M}$ is symmetric, i.e. $\frac{1}{2} \notin K$.

Proof. We first show that we may assume that

$$
M(0)=\left(\begin{array}{cccc}
0 & -1 & 0 & \ldots \\
1 & 0 & 0 & \ldots \\
0 & 0 & 0 & \ldots \\
\vdots & \vdots & \vdots & \vdots: \vdots
\end{array}\right)
$$

Notice that this is indeed the case if $\operatorname{rk} M(0) \geq 2$. Otherwise, we can follow the proof of theorem 2.1, to deduce that we may assume that $\operatorname{rk} M(0)=1$. So we may assume that

$$
M(0)=\left(\begin{array}{cccc}
0 & 0 & 0 & \ldots \\
1 & 0 & 0 & \ldots \\
0 & 0 & 0 & \ldots \\
\vdots & \vdots & \vdots & \vdots: \vdots
\end{array}\right)
$$


Suppose that an $\tilde{M}$ as in (3) cannot be obtained by interchanging the first and the second row of $M$. Then $M$ has a nonzero entry outside the second row and the first column. Without loss of generality, we may assume that the second entry of the first row of $M$ is nonzero.

Say that the coefficient of $x_{j}$ of this entry of $M$ is nonzero. Let $C$ be the coefficient matrix of $x_{j}$ of $M$. Then

$$
x_{j}^{-1}\left(M-x_{j} C\right)+x_{j}^{0} C
$$

is the expansion of the matrix $x_{j}^{-1} M$ to powers of $x_{j}$ (which may be negative), because $M-x_{j} C$ and $C$ are the coefficient matrices of $x_{j}^{-1}$ and $x_{j}^{0}$ of $x_{j}^{-1} M$ respectively. Let $C^{*}$ be the matrix one gets by substituting $x_{j}=x_{j}^{-1}$ in $x_{j}^{-1} M$. Then

$$
C^{*}=x_{j}^{+1}\left(M-x_{j} C\right)+x_{j}^{0} C=x_{j}\left(M-M(0)-x_{j} C\right)+x_{j} M(0)+C
$$

If $\operatorname{rk} C \geq 2$, then we can interchange $C$ and $M(0)$ as coefficient matrices of $M$ without affecting $\mathrm{rk} M=2$, namely by replacing $M$ by the result of substituting $x_{i}=x_{j}^{-1} x_{i}$ for all $i \neq j$ in $C^{*}$, to obtain $\operatorname{rk} M(0) \geq 2$ as above.

So assume that $\operatorname{rk} C=1$. Since the second entry of the first row of $C$ is nonzero, we may assume that the first row of $C$ equals $\left(\begin{array}{llllll}0 & 1 & 0 & 0 & \cdots & 0\end{array}\right)$. Since $\operatorname{rk} C=1$, it follows that only the second column of $C$ is nonzero, so we may assume that $C$ is the transpose of $M(0)$. Now replace $M$ by the result of substituting $x_{j}=x_{j}-1$ in $M$, to obtain that $M(0)$ is as in (2.1).

So $M(0)$ is as in (2.1). Looking at the linear parts of the $3 \times 3$ minor determinants, we see that only the first two rows and the first two columns of $M$ may be nonzero. Take $i \geq 3$ arbitrary.

Suppose that (2) does not hold. Then we may assume that the third row of $M$ is nonzero. Suppose first that the first entry of the third row of $M$ is zero. Then the second entry of the third row cannot be zero. Now we can replace $M$ by the result of adding the second column and the second row to the first column and the first row respectively in $M$, to make the first entry of the third row of $M$ nonzero as well, without affecting $M(0)$.

So we may assume that the first entry of the third row of $M$ is nonzero. Looking at the quadratic part of the leading principal $3 \times 3$ minor determinant, we see that the third column is dependent on the transpose of the third row. If $i>3$, then we could interchange the third and the $i^{\text {th }}$ column of $M$, so the $i^{\text {th }}$ column of $M$ is dependent on the transpose of the third row.

Suppose that (1) does not hold. Then we may assume that the third column of $M$ is nonzero. Just like the $i^{\text {th }}$ column of $M$ is dependent on the transpose of the third row, we can deduce that the $i^{\text {th }}$ row of $M$ is dependent on the transpose of the third column. So the $i^{\text {th }}$ row of $M$ is dependent on the third row. In addition, the $i^{\text {th }}$ column of $M$ is dependent on the third column.

Since the third column is dependent on the transpose of the third row, the third entry of the first row of $M$ is nonzero along with the first entry of the third row. Furthermore, we may assume that the leading principal $3 \times 3$ matrix 
of $M$ is of the form

$$
\left(\begin{array}{ccc}
* & * & -a \\
* & c & -b \\
a & b & 0
\end{array}\right) \quad \text { or } \quad\left(\begin{array}{ccc}
* & * & b \\
* & c & \lambda b \\
a & \lambda a & 0
\end{array}\right)
$$

where $a, b$ and $c$ are linear forms over $K$ and $\lambda \in K$.

Suppose first that the leading principal $3 \times 3$ matrix of $M$ is of the form of the rightmost matrix of (2.2). Then we can replace $M$ by the result of subtracting the first column and the first row $\lambda$ times from the second column and the second row respectively in $M$, to obtain $\lambda=0$ in the rightmost matrix of (2.2), without affecting $M(0)$. After that, we can look at the leading principal $3 \times 3$ minor determinant to deduce that $c$ has become zero. So (3) is satisfied.

Suppose next that the leading principal $3 \times 3$ matrix of $M$ is of the form of the leftmost matrix of (2.2), but not of the form of the rightmost matrix of (2.2). Then $a$ and $b$ are independent linear forms. Looking at the leading principal $3 \times 3$ minor determinant, we see that $b \mid a^{2} c$, so $b \mid c$ and $c=\mu b$ for some $\mu \in K$. Now replace $M$ by the result of subtracting the third row $\mu$ times from the second row, to obtain $\mu=0$, without affecting $M(0)$. So we may assume that $c=0$ in the leftmost matrix of (2.2). In a similar manner, we can clean the upper left corner of $M$, so we may assume that the diagonal of the leftmost matrix of (2.2) is zero.

Now it is straightforward to check that the leading principal $3 \times 3$ matrix of $M$ is antisymmetric. Furthermore, the three entries below its diagonal are linearly independent over $K$, because $a, b$ and $f+1$ are linearly independent over $K$ for every linear form $f$. Since $a$ and $b$ are linearly independent, it follows that the $i^{\text {th }}$ row of $M$ is dependent over $K$ on the third row. Since $-a$ and $-b$ are linearly independent, it follows that the $i^{\text {th }}$ column of $M$ is dependent over $K$ on the third column. So we can make $\tilde{M}$ as in (4) from $M$ by way of row and column operations.

So we have proved the first part of this theorem. To prove the second part of this theorem, assume that $M=\mathcal{J} H$ for a polynomial map $H$. Just as in the proof of theorem 2.1, we may assume that $H(0)=0$ and $\operatorname{deg} H \leq 2$. Say that $\tilde{M}=S M T$ for invertible matrices $S, T$ over $K$, and let $\tilde{H}:=S H(T x)$. The case where $\tilde{M}$ is as in (3) follows in a similar manner as the case where $\tilde{M}$ is as in (1) in the proof of theorem 2.1.

Hence assume that $\tilde{M}$ is as in (4) and that $\frac{1}{2} \in K$. Then $\mathcal{J} \tilde{H}$ is as in (4) as well, and $\operatorname{deg} \tilde{H} \leq 2$. From lemma 2.2 it follows that $\operatorname{deg} \tilde{H}=1$. This contradicts that $\tilde{M}$ has three entries which are linearly independent over $K$.

Corollary 2.4. Let $H$ be a quadratic polynomial map over $K$, such that $r:=$ rk $\mathcal{J} H \leq 2$. If $\frac{1}{2} \in K$, then $K[H] \subseteq K\left[f_{1}, \ldots, f_{r}\right]$ for polynomials $f_{i}$. In particular, rk $\mathcal{J} H=\operatorname{trdeg}_{K} K(H)$.

Proof. From $\frac{1}{2} \in K$, it follows that for every term $t$ of $H$,

$$
\frac{\partial}{\partial x_{i}} t \neq 0 \Longleftrightarrow x_{i} \mid t
$$


If $r=0$, then $H$ is constant. If $r=1$, then it follows from theorem 2.1 that we may assume that either $K[H]=K\left[x_{1}\right]$ or $K[H]=K\left[H_{1}\right]$. So assume that $r=2$. Then it follows from theorem 2.3 that we may assume that either $K[H] \subseteq K\left[x_{1}, x_{2}\right]$ or $K[H]=K\left[H_{1}, H_{2}\right]$ or $K[H]=K\left[H_{1}, x_{1}\right]$, because (4) of theorem 2.3 for $M=\mathcal{J} H$ requires $\frac{1}{2} \notin K$.

Lemma 2.5. Let $H$ be a polynomial map over $K$ and suppose that $\mathcal{J} H$ is symmetric. If for each $i$, the $i^{\text {th }}$ entry of the diagonal of $\mathcal{J} H$ has no terms whose degrees with respect to $x_{i}$ are equal to -2 in $K$, then there exists a polynomial $h \in K[x]$ such that $H=(\mathcal{J} h)^{\mathrm{t}}$ and $\mathcal{J} H=\mathcal{H} h$.

Proof. Assume that the diagonal of $\mathcal{J} H$ is as indicated above. Then for each $i$, $H_{i}$ has no terms whose degrees with respect to $x_{i}$ are equal to -1 in $K$. From the proof of $\mathrm{vdE}$, Lem. 1.3.53], it follows that there exists a polynomial $h \in K[x]$ such that $H=(\mathcal{J} h)^{\mathrm{t}}$ (the $\alpha_{i}$ in that proof are nonzero). So $\mathcal{J} H=\mathcal{H} h$.

Corollary 2.6. Suppose that $H$ is a polynomial map of degree at most 2 in dimension 3 over $K$, such that $\mathcal{J} H$ is antisymmetric with only zeroes on the diagonal. Suppose that $\mathcal{J} H$ is not constant. Then there exist $a \lambda \in K^{*}$ and $c_{1}, c_{2}, c_{3} \in K$, such that

$$
\mathcal{J} H=\mathcal{H}\left(\lambda\left(x_{1}+c_{1}\right)\left(x_{2}+c_{2}\right)\left(x_{3}+c_{3}\right)\right)
$$

Furthermore, $\frac{1}{2} \notin K$ and $\operatorname{rk} \mathcal{J} H=2<3=\operatorname{trdeg}_{K} K(H)$.

Proof. From lemma 2.2, it follows that $\frac{1}{2} \notin K$ and that $\mathcal{J} H$ is symmetric. From lemma 2.5, it follows that $\mathcal{J} H=\mathcal{H} h$ for some polynomial $h$.

Since $\operatorname{deg} H \leq 2$, it follows that terms of degree greater than 3 of $h$ cannot affect $\mathcal{H} h$. Since $\frac{1}{2} \notin K$, it follows that terms of degree at most 3 of $h$ which are divisible by $x_{i}^{2}$ for some $i$ cannot affect $\mathcal{H} h$. So we can remove terms of $h$ of degree greater than 3 and terms of $h$ which are divisible by $x_{i}^{2}$ for some $i$. Furthermore, we can remove terms of $h$ of degree at most 1. After these removals, $h$ will be of the form

$$
h=\lambda x_{1} x_{2} x_{3}+\tilde{c}_{1} x_{2} x_{3}+\tilde{c}_{2} x_{3} x_{1}+\tilde{c}_{3} x_{1} x_{2}
$$

In particular $\operatorname{deg} h \leq 3$. Suppose that $\mathcal{J} H$ is not constant. Then $\operatorname{deg} h=3$, so $\lambda \neq 0$. Hence $\mathcal{J} H$ is of the given form, with $c_{i}=\lambda^{-1} \tilde{c}_{i}$ for each $i$. Furthermore, rk $\mathcal{J} H=2$.

Suppose that $\operatorname{trdeg}_{K} K(H) \leq 2$. Then there exists a polynomial $f$ such that $f(H)=0$. If $\bar{f}$ is the leading homogeneous part of $f$ and $\bar{H}$ is the leading homogeneous part of $H$, then $\bar{f}(\bar{H})=0$, so $\operatorname{trdeg}_{K} K(\bar{H}) \leq 2$. From dB3, Th. 2.7], it follows that there exists an $S \in \mathrm{GL}_{3}(K)$ such that

$$
S \bar{H}=(p, q, 0) \quad \text { or } \quad S \bar{H}=\left(p^{2}, p q, q^{2}\right)
$$

for homogeneous polynomials $p, q$ of the same degree. In the first case, the rows of $\mathcal{J} \bar{H}$ are dependent over $K$. In the second case, $\operatorname{deg}(p, q)=1$ and the columns 
of $\mathcal{J} \bar{H}$ are dependent over $K$. This is however not the case for

$$
\mathcal{J} \bar{H}=\lambda\left(\begin{array}{ccc}
0 & x_{3} & x_{2} \\
x_{3} & 0 & x_{1} \\
x_{2} & x_{1} & 0
\end{array}\right)
$$

so $\operatorname{trdeg}_{K} K(H)=3$.

Corollary 2.7. Let $M$ be a matrix whose entries are polynomials of degree at most 1 over $K$. Suppose that $\mathrm{rk} M \leq 2$ and that $M$ is the Jacobian matrix of a polynomial map $H$. Then it is impossible to choose $H$ such that $\mathrm{rk} \mathcal{J} H=$ $\operatorname{trdeg}_{K} K(H)$ (and $\mathcal{J} H=M$ ), if and only if $M$ is as in (4) of theorem 2.3, In that case, $\frac{1}{2} \notin K$ and $\operatorname{rk} \mathcal{J} H=2<3=\operatorname{trdeg}_{K} K(H)$ for every polynomial map $H$ such that $\mathcal{J} H=M$.

Proof. The 'if'-part follows from corollary 2.6. The last claim follows from corollary 2.6 as well. The 'only if'-part follows from theorem 2.3 and the proof of corollary 2.4.

\section{Nilpotent Jacobian matrices of degree one and rank at most two}

Before we prove the main result of this section, which is theorem 3.2 below, we formulate a lemma about nilpotent matrices $N$ of degree 1 and size $2 \times 2$ or $3 \times 3$, for which $N(0)$ has a simple structure.

Lemma 3.1. Let $K$ be a field and $N$ be a nilpotent matrix whose entries are polynomials of degree 1 . Then the following holds.

(i) If

$$
N(0)=\left(\begin{array}{ll}
0 & 0 \\
0 & 0
\end{array}\right)
$$

then $N$ is similar over $K$ to a triangular matrix with only zeroes on the diagonal.

(ii) If

$$
N(0)=\left(\begin{array}{ll}
0 & 0 \\
1 & 0
\end{array}\right)
$$

then $N$ is lower triangular with only zeroes on the diagonal.

(iii) If

$$
N(0)=\left(\begin{array}{lll}
0 & 0 & 1 \\
0 & 0 & 0 \\
0 & 0 & 0
\end{array}\right)
$$

then $N$ is similar over $K$ to a triangular matrix with only zeroes on the diagonal. 
(iv) If

$$
N(0)=\left(\begin{array}{lll}
0 & 1 & 0 \\
0 & 0 & 1 \\
0 & 0 & 0
\end{array}\right)
$$

and $N$ is not upper triangular, then $N$ is similar over $K$ to a matrix of the form

$$
\left(\begin{array}{ccc}
0 & f+1 & 0 \\
b & 0 & f+1 \\
0 & -b & 0
\end{array}\right)
$$

where $f$ and $b$ are linear forms and $b \neq 0$.

Proof. Since $N$ is nilpotent, it follows from lemma 1.5 that for every $r \leq n$, the sum of the principal minor determinants of size $r \times r$ is zero. This gives us the trace condition if $r=1$, the $2 \times 2$ principal minors condition if $r=2$, and the determinant condition if $r=n$.

(i) Using the trace condition, we see that

$$
N=\left(\begin{array}{cc}
a & b \\
c & -a
\end{array}\right)
$$

for linear forms $a, b$. Using the determinant condition, we see that $b c=a^{2}$, so $b \mid a^{2}$. As polynomial rings have unique factorization, $b=\lambda a$ for some $\lambda \in K$. Since $c=\lambda^{-1} a$, the entries of $a^{-1} N$ are contained in $K$. Hence $a^{-1} N$ is strongly nilpotent over an infinite extension field of $K$. From proposition 1.3, it follows that $a^{-1} N$ is similar over $K$ to a triangular matrix with only zeroes on the diagonal, and so is $N$.

(ii) Using the determinant condition,

$$
N=\left(\begin{array}{ll}
* & 0 \\
* & *
\end{array}\right)
$$

(because the linear part is zero). Hence $N$ is lower triangular. From lemma 1.2, it follows that $N$ has only zeroes on the diagonal.

(iii) Using the principal $2 \times 2$ minors condition, we see that

$$
N=\left(\begin{array}{lll}
* & * & * \\
b & * & * \\
0 & c & *
\end{array}\right)
$$

for linear forms $b, c$ (because the linear part is zero). On account of the determinant condition, $b c=0$ (because $b c$ is the quadratic part of $\operatorname{det} N$ ).

If $b=0$, then the leading principal $1 \times 1$ minor matrix and the trailing principal $2 \times 2$ minor matrix of $N$ are nilpotent on account of lemma 1.2 If $c=0$, then the leading principal $2 \times 2$ minor matrix and the trailing principal $1 \times 1$ minor matrix of $N$ are nilpotent on account of lemma 1.2 
In both cases, the principal $1 \times 1$ minor matrix is zero, because $\operatorname{Mat}_{1}(K)$ is a reduced ring. From (i), we deduce that in both cases, the principal $2 \times 2$ minor matrix is similar over $K$ to a triangular matrix with only zeroes on the diagonal. On account of corollary 1.4, $N$ is similar over $K$ to a triangular matrix with only zeroes on the diagonal.

(iv) Using all principal minors conditions

$$
N=\left(\begin{array}{ccc}
-a & * & * \\
b & a+c & * \\
0 & -b & -c
\end{array}\right)
$$

for linear forms $a, b, c$ (because the linear parts are zero). If $b=0$, then $N$ is upper triangular, so assume that $b \neq 0$. On account of the determinant condition, $a=c$ (quadratic part) and $b \mid a(a+c) c$ (cubic part). So $b \mid a$ if $\frac{1}{2} \in K$. If $\frac{1}{2} \notin K$, then $b \mid a^{2}$ on account of the principal $2 \times 2$ minors condition, so $b \mid a$ in any case.

Since $b \mid a$ and $b \mid c$, we may assume that $a=c=0$, because we can replace $N$ by $T^{-1} N T$, where

$$
T:=\left(\begin{array}{ccc}
1 & -\frac{a}{b} & 0 \\
0 & 1 & -\frac{c}{b} \\
0 & 0 & 1
\end{array}\right) \quad \text { and } \quad T^{-1}=\left(\begin{array}{ccc}
1 & \frac{a}{b} & \frac{a c}{b^{2}} \\
0 & 1 & \frac{c}{b} \\
0 & 0 & 1
\end{array}\right)
$$

On account of the determinant condition, the upper right corner of $N$ is zero. On account of the principal $2 \times 2$ minors condition, $N$ is of the form of (3.1) for some linear form $f$.

A square matrix over $K$ is similar over $K$ to its so-called Jordan normal form, if and only if all its eigenvalues are contained in $K$. Hence it follows from lemma 1.5 that every nilpotent matrix over $K$ is similar over $K$ to its Jordan normal form.

This fact about Jordan normal forms is used in the proof of theorem 3.2 below. The reader who is not familiar with Jordan normal forms has to show some required similarities by hand, using the result of proposition 1.3 that nilpotent matrices over $K$ are similar over $K$ to triangular matrices with zeroes on their diagonals.

Theorem 3.2. Suppose that $H$ is a quadratic polynomial map in dimension $n$ over a field $K$ of any characteristic, such that $\mathrm{rk} \mathcal{J} H \leq 2$ and $\mathcal{J} H$ is nilpotent. Then $\mathcal{J} H$ is similar over $K$ to a triangular matrix.

Proof. Let $M=\mathcal{J} H$. Suppose first that $\operatorname{rk} M=1$. From theorem 2.1, it follows that there exists $S, T \in \mathrm{GL}_{n}(K)$, such that $\tilde{M}:=S M T$ satisfies one of the following:

- $\tilde{M}$ is as in (1) of theorem 2.1.

Then $\tilde{M}$ and $T^{-1} M T$ are lower triangular, because only their first columns are nonzero. So $M$ is similar over $K$ to a triangular matrix. 
- $\tilde{M}$ is as in (2) of theorem 2.1.

Then $\tilde{M}$ and $S M S^{-1}$ are upper triangular, because only their first rows are nonzero. So $M$ is similar over $K$ to a triangular matrix.

Suppose next that $\mathrm{rk} M=2$. From theorem [2.3, it follows that there exists $S, T \in \mathrm{GL}_{n}(K)$, such that $\tilde{M}:=S M T$ satisfies one of the following:

- $\tilde{M}$ is as in (1) of theorem 2.3 .

Then only the first two columns of $\tilde{M}$ and $T^{-1} M T$ are nonzero. On account of lemma 1.2, the leading principal $2 \times 2$ minor matrix $N$ of $T^{-1} M T$ is nilpotent.

Since the Jordan normal form of $N(0)$ is equal to (that of) $N(0)$ in (i) or (ii) of lemma 3.1, it follows from (i) and (ii) of lemma 3.1 that $N$ is similar over $K$ to a triangular matrix with only zeroes on the diagonal. From corollary 1.4, we deduce that $M$ is similar over $K$ to a triangular matrix as well.

- Only the first three rows of $\tilde{M}$ may be nonzero.

Then only the first three rows of $\tilde{M}, S M S^{-1}$ and $\mathcal{J}\left(S H\left(S^{-1} x\right)\right)=$ $\left.S M\right|_{x=S^{-1} x} S^{-1}$ are nonzero. On account of lemma 1.2, the leading principal $3 \times 3$ minor matrix $N$ of $S M S^{-1}$ is nilpotent.

In order to show that $M$ is similar over $K$ to a triangular matrix, it suffices to show that $S M S^{-1}$ is similar over $K$ to a triangular matrix. From corollary 1.4, we deduce that it suffices to show that $N$ is similar over $K$ to a triangular matrix with only zeroes on the diagonal. For that purpose, we distinguish three cases.

$\operatorname{rk} \boldsymbol{N}(\mathbf{0})=\mathbf{0}$. Then we can replace $M$ by the result of substituting $x_{i}=$ $x_{i}+1$ in $M$ for some $i$, to obtain $\operatorname{rk} N(0) \neq 0$, because of the following. $M$ becomes $\mathcal{J}\left(\left.H\right|_{x_{i}=x_{i}+1}\right)$, which is a Jacobian matrix as well, and the linear part of $N$ is not affected.

So if $N$ is similar over $K$ to a triangular matrix with only zeroes on the diagonal in the new situation, then $N$ is similar over $K$ to a triangular matrix with only zeroes on the diagonal originally.

$\operatorname{rk} N(\mathbf{0})=\mathbf{1}$. Then the Jordan normal form of $N(0)$ is equal to that of $N(0)$ in (iii) of lemma 3.1. So we can choose $S$, such that $N(0)$ is as in (iii) of lemma 3.1 It follows from (iii) of lemma 3.1 that $N$ is similar over $K$ to a triangular matrix with only zeroes on the diagonal.

$\operatorname{rk} \boldsymbol{N}(\mathbf{0}) \geq \mathbf{2}$. Then the Jordan normal form of $N(0)$ is equal to (that of) $N(0)$ in (iv) of lemma 3.1. So we can choose $S$, such that $N(0)$ is as in (iv) of lemma 3.1 .

Suppose first that $N$ is upper triangular. From lemma 1.2, it follows that the leading principal minor matrix of size $1 \times 1$ and the trailing principal minor matrix of size $2 \times 2$ of $N$ are nilpotent. By applying lemma 1.2 on the trailing principal minor matrix of size $2 \times 2$, we 
see that every principal minor matrix of size $1 \times 1$ is nilpotent. As $\operatorname{Mat}_{1}(K)$ is a reduced ring, the diagonal of $N$ is totally zero.

So assume that $N$ is not upper triangular. Then it follows from (iv) of lemma 3.1 that we can choose $S$, such that $N$ is of the form of (3.1), where $f$ and $b$ are linear forms and $b \neq 0$, because $N(0)$ will not be affected. Define

$$
\tilde{N}:=N \cdot\left(\begin{array}{ccc}
0 & 0 & -1 \\
0 & -1 & 0 \\
1 & 0 & 0
\end{array}\right)=\left(\begin{array}{ccc}
0 & -f-1 & 0 \\
f+1 & 0 & -b \\
0 & b & 0
\end{array}\right)
$$

Notice that $\tilde{N}$ is antisymmetric. By definition of $\tilde{N}$ and $\tilde{M}$, we can choose $T$ such that $\tilde{N}$ is the leading principal $3 \times 3$ minor matrix of $\tilde{M}$.

Since the leading principal $2 \times 2$ minor matrix of $\tilde{N}(0)$ has full rank, we can clean the parts outside $\tilde{N}(0)$ of the first two rows of $\tilde{M}(0)$ by way of column operations. In other words, we can choose $T$ such that the submatrix of the first two rows of $\tilde{M}(0)$ equals

$$
\left(\begin{array}{ccc|ccc}
0 & -1 & 0 & 0 & \cdots & 0 \\
1 & 0 & 0 & 0 & \cdots & 0
\end{array}\right)
$$

Looking at the constant parts of the $3 \times 3$ minor determinants, we see that the third and subsequent entries of the third row of $\tilde{M}(0)$ are zero.

Looking at the linear parts of the $3 \times 3$ minor determinants, we see that the second entry of the third row of $\tilde{M}$ is the only nonzero entry in that row. So the third row of $\tilde{M}$ is of the form

$$
\left(\begin{array}{lll|lll}
0 & b & 0 & 0 & \cdots & 0
\end{array}\right)
$$

On account of (1.2), $\mathcal{J} \tilde{H}=\left.\tilde{M}\right|_{x=T x}$. Hence the second entry $\tilde{b}$ of the third row of $\mathcal{J} \tilde{H}$ is the only nonzero entry in that row. Furthermore, $\tilde{b}$ is a nonzero linear form just like $b$.

Consequently, $\tilde{b}=\lambda x_{2}$ for some nonzero $\lambda \in K$. So the coefficient of $x_{2}^{2}$ in $\tilde{H}_{3}$ equals $\frac{1}{2} \lambda$. In particular,

$$
\frac{1}{2} \in K \quad \text { and } \quad \operatorname{deg}_{x_{2}} \tilde{H}_{3}>1
$$

Since $\mathcal{J}_{x_{1}, x_{2}, x_{3}}\left(\tilde{H}_{1}, \tilde{H}_{2}, \tilde{H}_{3}\right)=\left.\tilde{N}\right|_{x=T x}$ is antisymmetric, it follows from lemma 2.2 that

$$
\operatorname{deg}_{x_{2}} \tilde{H}_{3} \leq \operatorname{deg}_{x_{1}, x_{2}, x_{3}}\left(\tilde{H}_{1}, \tilde{H}_{2}, \tilde{H}_{3}\right) \leq 1
$$

Contradiction, so $N$ is upper triangular. 
In the proof of [PC, Lem. 4], it is shown that $\mathcal{J} H^{2}=0$ implies $(\mathcal{J} H)(x)$. $(\mathcal{J} H)(y)=0$ if $H$ is quadratic homogeneous and $\frac{1}{2} \in K$, where $y=\left(y_{1}, y_{2}, \ldots\right.$, $\left.y_{n}\right)$ is another $n$-tuple of indeterminates. The maps

$$
H=\left(0, x_{1}, x_{1}^{2}, x_{1} x_{2}-\frac{1}{2} x_{3}\right)
$$

and

$$
H=\left(0,0,0, x_{2} x_{3}, x_{3} x_{1}, x_{1} x_{2}, x_{1} x_{4}+x_{2} x_{5}+x_{3} x_{6}\right)
$$

show that the conditions that $H$ is (quadratic) homogeneous and $\frac{1}{2} \in K$ are necessary respectively.

Theorem 3.3. Suppose that $H$ is a quadratic polynomial map in dimension $n$ over a field $K$ of any characteristic, such that $\mathcal{J} H^{2}=0$. Then the following holds.

(i) $\mathcal{J} H$ is similar over $K$ to a triangular matrix.

(ii) If $\frac{1}{2} \in K$ and $H$ is homogeneous, then

$$
(\mathcal{J} H)(x) \cdot(\mathcal{J} H)(y)=0
$$

where $y=\left(y_{1}, y_{2}, \ldots, y_{n}\right)$ is another $n$-tuple of indeterminates.

(iii) If $\frac{1}{2} \in K$ and $H$ is not (necessarily) homogeneous, then

$$
(\mathcal{J} H)(x) \cdot(\mathcal{J} H)(y) \cdot(\mathcal{J} H)(z)=0
$$

where $z=\left(z_{1}, z_{2}, \ldots, z_{n}\right)$ is yet another $n$-tuple of indeterminates.

Proof. Let $y=\left(y_{1}, y_{2}, \ldots, y_{n}\right)$ be an $n$-tuple of indeterminates. From $\operatorname{deg} \mathcal{J} H \leq$ 1 , it follows that

$$
(\mathcal{J} H)(t x+(1-t) y)=t(\mathcal{J} H)(x)+(1-t)(\mathcal{J} H)(y)
$$

Taking squares on both sides, we deduce that

$$
0=t(1-t)((\mathcal{J} H)(x) \cdot(\mathcal{J} H)(y)+(\mathcal{J} H)(y) \cdot(\mathcal{J} H)(x))
$$

Consequently,

$$
(\mathcal{J} H)(y) \cdot(\mathcal{J} H)(x)=-(\mathcal{J} H)(x) \cdot(\mathcal{J} H)(y)
$$

(i) Let

$$
Z=\left(\begin{array}{cccc}
Z_{11} & Z_{12} & \cdots & Z_{1 n} \\
Z_{21} & Z_{22} & \cdots & Z_{2 n} \\
\vdots & \vdots & \vdots \vdots & \vdots \\
Z_{n 1} & Z_{n 2} & \cdots & Z_{n n}
\end{array}\right)
$$


be a matrix of indeterminates. Since we can substitute elements of any field $L \supseteq K$ in the indeterminates of the matrix $(Z|x| y)$, it follows from proposition 1.3 that it suffices to show that

$$
\left(\prod_{i=1}^{n}(\mathcal{J} H)\left(Z e_{i}\right)\right) \cdot(\mathcal{J} H)(x) \cdot(\mathcal{J} H)(y)=0
$$

Just as in the proof of (iii) below, we can reduce to the case where $H$ is homogeneous, at the cost of losing $(\mathcal{J} H)(y)$ as a factor. So if we show that

$$
\left(\prod_{i=1}^{n}(\mathcal{J} H)\left(Z e_{i}\right)\right) \cdot(\mathcal{J} H)(x)=0
$$

then we may assume that $H$ is homogeneous.

Hence suppose that $H$ is homogeneous. From (ii) of [PC, Prop. 3] and (3.2), it follows that

$$
\left(\prod_{i=1}^{n}(\mathcal{J} H)\left(Z e_{i}\right)\right) \cdot(\mathcal{J} H)(x) \cdot Z e_{j}=(\vdots \vdots: \vdots) \cdot\left((\mathcal{J} H)\left(Z e_{j}\right)\right)^{2} \cdot x=0
$$

for every $j \leq n$. Consequently

$$
\left(\prod_{i=1}^{n}(\mathcal{J} H)\left(Z e_{i}\right)\right) \cdot(\mathcal{J} H)(x) \cdot Z=0
$$

As $\operatorname{rk} Z=n,(3.3)$ follows.

(ii) This is shown in the proof of [PC, Lem. 4].

(iii) Let $\bar{H}$ be the quadratic part of $H$. Notice that

$$
\prod_{i=1}^{3}(\mathcal{J} H)\left(Z e_{i}\right)=\prod_{i=1}^{3}\left((\mathcal{J} \bar{H})\left(Z e_{i}\right)+(\mathcal{J} H)(0)\right)
$$

and that every term of the expansion of the right hand side of (3.4) either has two factors $(\mathcal{J} H)(0)$, or two distinct (but not necessarily different) factors $(\mathcal{J} \bar{H})\left(Z e_{i}\right)$ and $(\mathcal{J} \bar{H})\left(Z e_{j}\right)$, where $1 \leq i \leq j \leq 3$.

From (3.2), it follows that

$$
\begin{aligned}
((\mathcal{J} \bar{H})(x))^{2} & =((\mathcal{J} H)(x)-(\mathcal{J} H)(0))^{2} \\
& =((\mathcal{J} H)(x))^{2}+((\mathcal{J} H)(0))^{2}=0
\end{aligned}
$$

From (ii), we subsequently deduce that

$$
(\mathcal{J} \bar{H})(x) \cdot(\mathcal{J} \bar{H})(y)=0
$$


Furthermore, it follows from (3.2) that

$$
\begin{aligned}
(\mathcal{J} \bar{H})(x) \cdot(\mathcal{J} H)(0) & =((\mathcal{J} H)(x)-(\mathcal{J} H)(0)) \cdot(\mathcal{J} H)(0) \\
& =-(\mathcal{J} H)(0) \cdot((\mathcal{J} H)(x)-(\mathcal{J} H)(0)) \\
& =-(\mathcal{J} H)(0) \cdot(\mathcal{J} \bar{H})(x)
\end{aligned}
$$

Consequently, the factors of the terms of the expansion of the right hand side of (3.4) anticommute. From (3.5) and $((\mathcal{J} H)(0))^{2}=0$, we deduce that every term of the expansion of the right hand side of (3.4) equals zero. So $(\mathcal{J} H)\left(Z e_{1}\right) \cdot(\mathcal{J} H)\left(Z e_{2}\right) \cdot(\mathcal{J} H)\left(Z e_{3}\right)=0$.

The conclusions of (ii) and (iii) of theorem 3.3 can be reformulated as properties of a triangular matrix to which $J H$ is similar over $K$, see [dB1, Th. 2.1] and $\mathrm{dB} 1$, Cor. 2.2]. Using this reformulation more generally, one can deduce the following.

Proposition 3.4. Let $H$ be a polynomial map, such that $\mathcal{J} H$ is similar over $K$ to a triangular matrix. If $\mathcal{J} H$ is nilpotent and $r=\operatorname{rk} \mathcal{J} H$, then

$$
(\mathcal{J} H)\left(Z e_{1}\right) \cdot(\mathcal{J} H)\left(Z e_{2}\right) \cdots(\mathcal{J} H)\left(Z e_{r}\right) \cdot(\mathcal{J} H)\left(Z e_{r+1}\right)=0
$$

where $Z$ is as in the proof of (i) of theorem 3.3.

It follows that the conclusions of (ii) and (iii) of theorem 3.3 can be added to the cases rk $\mathcal{J} H=1$ and $\mathrm{rk} \mathcal{J} H=2$ of theorem 3.2 respectively as well.

\section{References}

[dB1] Michiel de Bondt. The strong nilpotency index of a matrix. Linear Multilinear Algebra, 62(4):486-497, 2014.

[dB2] Michiel de Bondt. Symmetric Jacobians. Cent. Eur. J. Math., 12(6):787$800,2014$.

[dB3] Michiel de Bondt. Rational maps $H$ for which $K(t H)$ has transcendence degree 2 over $K$. arXiv:1501.06046, 2015.

[dBY] Michiel de Bondt and Dan Yan. Triangularization properties of power linear maps and the structural conjecture. Ann. Polon. Math., $112(3): 247-266,2014$.

[vdE] Arno van den Essen. Polynomial automorphisms and the Jacobian conjecture, volume 190 of Progress in Mathematics. Birkhäuser Verlag, Basel, 2000.

[FMN] Piotr Faliszewski, Anca Muscholl, and Rolf Niedermeier, editors. 41st International Symposium on Mathematical Foundations of Computer Science, MFCS 2016, August 22-26, 2016 - Kraków, Poland, volume 58 of LIPIcs. Schloss Dagstuhl - Leibniz-Zentrum fuer Informatik, 2016. 
[MO] Gary H. Meisters and Czesław Olech. Strong nilpotence holds in dimensions up to five only. Linear and Multilinear Algebra, 30(4):231-255, 1991.

[PSS] Anurag Pandey, Nitin Saxena, and Amit Sinhababu. Algebraic independence over positive characteristic: New criterion and applications to locally low algebraic rank circuits. In Faliszewski et al. [FMN], pages 74:1-74:15.

[PC] Kevin Pate and Charles Ching-An Cheng. Quadratic homogeneous Keller maps of rank two. Linear Algebra Appl., 476:16-27, 2015. 